\section{Comment on Gialluisi et al}

\author{
European Journal of Human Genetics (2014) 22, 157; \\ doi:10.1038/ejhg.2013.152; published online 17 July 2013
}

As authors of the first paper describing the methodology used by Gialluisi et al in their paper on the high allele frequency for Wilson disease in the Sardinian population, we want to congratulate them with their result. ${ }^{1,2}$ The paper clearly shows the strength of this methodology. We were also particularly impressed by their conscientious approach to determine the inbreeding coefficient in this special population.

Still we want to draw attention to some inaccuracies in their paper, which-in our opinion-should be avoided by future users of this method, as they may result in underestimation of the gene frequency - ie, the total pathogenic allele frequency-and birth prevalence of the disorder. First of all, the authors disregard 14 mutations with relative frequencies below $1 \%$. By including these, the gene frequency becomes 0.0195 , instead of 0.0191 . Second, the authors included only patients with unambiguous genotype and detailed geographical provenance of parents. However, leaving out seemingly heterozygous patients with a second, unidentified mutation will have the same effect on the estimation of the gene frequency as disregarding known mutations. Finally, the authors seem to have calculated the birth frequency of the disorder in Sardinia by simply squaring the gene frequency. This can be justified in random mating populations but not in the Sardinian population where the inbreeding coefficient is higher than zero. For instance in the mountains, where the inbreeding coefficient is 0.00112344 , and using the gene frequency estimate of Gialluisi et $a l^{2}$ the birth prevalence of Wilson disease will be 1:2585 instead of the 1:2732 calculated by these authors. Using the gene frequency estimate that includes the 14 rare mutations, the prevalence estimate in the mountains even becomes 1:2499, - 11\% higher than the original estimate.

We admit that each of our proposed corrections separately only has a small effect for the final estimate, but these effects are additive, and thus together not always insignificant. Therefore, we hope that by pointing to these inaccuracies future users of the methodology will be forewarned.

\section{CONFLICT OF INTEREST}

The authors declare no conflict of interest.

Leo P ten Kate, Marieke Teeuw, Lidewij Henneman and Martina C Cornel Department of Clinical Genetics, Institute of Health and Care Research, VU University Medical Center, Amsterdam, The Netherlands E-mail:lp.tenkate@vumc.nl

\footnotetext{
1 Ten Kate LP, Teeuw M, Henneman L, Cornel MC: Autosomal recessive disease in children of consanguineous parents: inferences from the proportion of compound heterozygotes. J Comm Genet 2010; 1: 37-40.

2 Gialluisi A, Incollu S, Pippucci T et al: The homozygosity index (HI) reveals high allele frequency for Wilson disease in the Sardinian population. Eur J Hum Genet 2013; 21: 1308-1311.
}

\section{Reply to ten Kate et al}

European Journal of Human Genetics (2014) 22, 157-158; doi:10.1038/ejhg.2013.153; published online 17 July 2013

We thank Prof ten Kate et $a l^{1}$ for the useful observations, which will allow us to clarify some aspects of the HI method., ${ }^{2,3}$

With regard to the inclusion of mutations with relative frequency below $1 \%$ in the formula, we may notice how these negligibly affect the final result, with a gene frequency increasing from 1.91 to $1.95 \%$. This corresponds to an even smaller increase in the prevalence of the disease (from $3.65 \times 10^{-4}$ to $3.8 \times 10^{-4}$, if we assume $P=q^{2}$ to simplify).

With reference to the exclusion of heterozygous patients from the formula, this is a conservative choice that we made under the basic assumption of strict recessive inheritance in the model, as including them would mean to (erroneously) assume that they are all compound heterozygotes. We also underline that, in this specific study, every sample was first investigated for the six most common reported mutations in Sardinia, and then, if no mutation was found, by single-strand conformation polymorphisms and Sanger sequencing of all exons and of the flanking intronic regions in the ATP7B gene. This makes it highly unlikely that a mutation already found in the Sardinian population was undetected.
Finally, we agree that in a non-random mating population $q \rightarrow P$ (instead of $P=q^{2}$, as per Hardy-Weinberg equilibrium). However, the current Sardinian population is characterized by high endogamy more than by high frequency of consanguineous matings. Under this scenario, also known as 'random inbreeding, ${ }^{4}$ the chance that an individual will mate with a genetically related one will be higher, and will finally lead to $P \approx q^{2}$, as already discussed in a recent letter published in this Journal. ${ }^{5}$

Nonetheless, we acknowledge that this is an unavoidable approximation and we always mentioned the gene frequency $(q)$ of a recessive disorder instead of the Prevalence $(P)$ as the main output of the HI method, inferring $P$ only when required for a comparison with other epidemiological methods. ${ }^{2,3}$

In summary, we agree with ten Kate et al ${ }^{1}$ that all of these details only slightly affect the final result but we disagree that they will jointly create a big discrepancy between our $q$ estimate and the real one, as also demonstrated by the factual equivalence between our $q$ and the one estimated by Zappu et $a l^{6}$ through a classical and reliable neonatal molecular screening. What is more important, the main purpose of the HI method is not to make a precise inference of the prevalence of a given recessive disorder in a population (as variables like the inbreeding coefficient can still be a source of error), but to produce 'a ranking order of the prevalence of autosomal recessive disorders, thus establishing priorities for genetic testing at the population level', 2,3

Our wish is that the HI method becomes of common use by public health institutions, especially in those country characterized by highly 
endogamous/consanguineous populations, and that it can be improved by making use of precise estimates of the inbreeding coefficient based on the analysis of genomic patterns of homozygosity. ${ }^{7}$

\section{CONFLICT OF INTEREST}

The authors declare no conflict of interest.

Alessandro Gialluisi ${ }^{1}$, Tommaso Pippucci ${ }^{2}$ and Giovanni Romeo ${ }^{\star, 2,3}$

${ }^{1}$ Language and Genetics Department, Max Planck Institute for Psycholinguistics, Nijmegen, The Netherlands;

${ }^{2}$ Unità Operativa di Genetica Medica, Policlinico Sant'Orsola Malpighi, Bologna, Italy;

${ }^{3}$ European Genetics Foundation, Bologna, Italy

E-mail: romeo@eurogene.org
1 Ten Kate LP, Teeuw M, Henneman L, Cornel MC: Comment on Gialluisi et al. Eur J Hum Genet 2014; 22: 157.

2 Gialluisi A, Pippucci T, Anikster $Y$ et al: Estimating the allele frequency of autosomal recessive disorders through mutational records and consanguinity: the homozygosity index (HI). Ann Hum Genet 2012; 76: 159-167.

3 Gialluisi A, Incollu S, Pippucci T et al: The homozygosity index (HI) approach reveals high allele frequency for Wilson disease in the Sardinian population. Eur J Hum Genet 2013; 21: 1308-1311.

4 Cavalli-Sforza LL, Moroni A, Zei G: Consanguinity, Inbreeding and Genetic Drift in Italy. Princeton, NJ: Princeton University Press, 2004.

5 Gialluisi A, Dediu D, Francks C, Fisher SE: Persistence and transmission of recessive deafness and sign language: new insights from village sign languages. Eur J Hum Genet 2013; 21: 894-896.

6 Zappu A, Magli O, Lepori MB et al: High incidence and allelic homogeneity of Wilson disease in 2 isolated populations: a prerequisite for efficient disease prevention programs. J Pediatr Gastroenterol Nutr 2008; 47: 334-338.

7 Pemberton TJ, Absher D, Feldman MW, Myers RM, Rosenberg NA, Li JZ: Genomic patterns of homozygosity in worldwide human populations. The Am J Hum Genet 2012; 91: 275-292.

\section{Reconciling clinical importance and statistical significance}

European Journal of Human Genetics (2014) 22, 158-159; doi:10.1038/ejhg.2013.110; published online 22 May 2013

Upon revisiting our published work on the genetic architecture of adult height, ${ }^{1}$ we noted the following sentence: 'Height was measured with a stadiometer to the nearest $0.1 \mathrm{~cm}$.' Genetic association studies generally do not utilize information regarding precision of phenotypic measurements. We suggest that doing so addresses a long-standing issue between clinical importance and statistical significance. In this specific instance, we suggest that effects smaller than $0.1 \mathrm{~cm}$ are clinically insignificant, as they are not measurable; the issue then becomes how to incorporate this limit into statistical approaches in human genetics investigations such as genome-wide association studies (GWAS).

One solution to this problem is based on classical measurement error. Assume that the observed value of the outcome (dependent variable) $y^{*}$ is equal to the true, underlying value of $y$ plus a random component $e$. In ordinary least squares (OLS), the true model $\boldsymbol{y}=\boldsymbol{X} \beta+\boldsymbol{\varepsilon}$ becomes $\boldsymbol{y}^{*}=\boldsymbol{X} \beta+\boldsymbol{e}+\boldsymbol{\varepsilon}$. Assuming that (1) the two errors are uncorrelated, (2) the expected values of both errors are 0 , and (3) both error terms are uncorrelated with the independent variable, the OLS estimate $\hat{\beta}$ is a consistent and unbiased estimate of $\beta$. However, the variance of $\hat{\beta}$ increases from $\left(\boldsymbol{X}^{\prime} \boldsymbol{X}\right)^{-1} \operatorname{var}(\boldsymbol{\varepsilon})$ to $\left(\boldsymbol{X}^{\prime} \boldsymbol{X}\right)^{-1}[\operatorname{var}(\boldsymbol{e})+\operatorname{var}(\boldsymbol{\varepsilon})]$. Consequently, test statistics accounting for measurement error will be smaller. Critically, claims of statistical significance will be limited by the precision of the measurement of the outcome.

A second solution to this problem is to use protected inference. ${ }^{2}$ Classical inference is based on testing what is called a point null hypothesis: in GWAS, a normally distributed test statistic can be formulated as $\left(\hat{\beta}-\beta_{0}\right) / \operatorname{SE}(\hat{\beta})$ with the point null value $\beta_{0}=0$. In slightly simplified terms, $\hat{\beta}$ is called consistent if it converges to the true value of $\beta$ with enough data, ie, a suitably large sample size. However, the probability mass of any point in a continuous distribution is 0 . Consequently, statistical significance can be attained for trivial effects by simply increasing the sample size (Figure 1a). Under protected inference, the null hypothesis is an interval rather than a point. We suggest two ways to implement protected inference. We can control the false positive error rate at the borders of the null interval, allowing the test to become overly conservative within the null interval (Figure 1b). Alternatively, we can control the false positive error rate at a fixed value across the entire null interval (Figure 1c). We recommend this latter approach, formulating the test statistic as $\left\{\begin{array}{cc}\frac{|\hat{\beta}|-\beta_{0}}{\operatorname{SE}(\hat{\beta})} & \text { if }|\hat{\beta}| \geq \beta_{0} \\ 0 & \text { otherwise }\end{array}\right.$, with $\beta_{0}$ now corresponding to the limit of precision, eg, $0.1 \mathrm{~cm}$ for our stadiometer. Either way, protected inference prevents arbitrarily large sample sizes from yielding statistical significance for trivial effects.
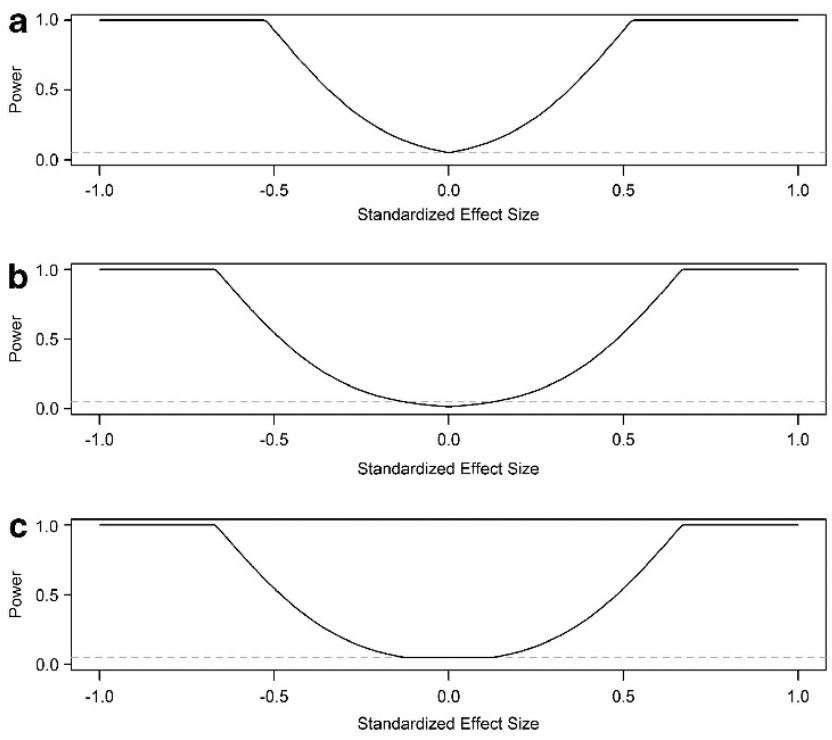

Figure 1 Power in classical vs protected inference. The gray dashed lines represent the significance level $\alpha=0.05$. (a) Classical inference involves testing the point null hypothesis that the effect size is 0 . (b) Protected inference involves testing a null interval hypothesis. In this implementation of protected inference, the false positive error rate is controlled at the borders of the null interval. (c) In this alternative implementation of protected inference, the false positive error rate is fixed across the entire null interval. 